Revue de l'Institut des langues et cultures

d'Europe, Amérique, Afrique, Asie et Australie

$34 \mid 2019$

Femmes et migrations aux $\mathrm{XIX}^{\mathrm{e}}$ et $\mathrm{XX} \mathrm{X}^{\mathrm{e}}$ siècles :

regards et représentations

\title{
L'émigration des femmes célibataires vers les colonies de l'Empire britannique de 1849 à 1865 : entre philanthropie, féminisme et colonisation matrimoniale
}

The Emigration of Single Women to the British Colonies between 1849 and 1865:

Philanthropy, Feminism and Matrimonial Colonisation

\section{Véronique Molinari}

\section{OpenEdition}

Journals

Édition électronique

URL : http://journals.openedition.org/ilcea/5657

DOI : 10.4000/ilcea.5657

ISSN : 2101-0609

Éditeur

UGA Éditions/Université Grenoble Alpes

Édition imprimée

ISBN : 978-2-37747-074-7

ISSN : 1639-6073

Référence électronique

Véronique Molinari, «L'émigration des femmes célibataires vers les colonies de l'Empire britannique

de 1849 à 1865 : entre philanthropie, féminisme et colonisation matrimoniale », ILCEA [En ligne],

34 | 2019, mis en ligne le 15 janvier 2019, consulté le 02 mai 2019. URL : http://

journals.openedition.org/ilcea/5657 ; DOI : 10.4000/ilcea.5657

Ce document a été généré automatiquement le 2 mai 2019.

(C) ILCEA 


\section{L'émigration des femmes célibataires vers les colonies de l'Empire britannique de 1849 à 1865 : entre philanthropie, féminisme et colonisation matrimoniale}

The Emigration of Single Women to the British Colonies between 1849 and 1865: Philanthropy, Feminism and Matrimonial Colonisation

Véronique Molinari

" The daughters of England are too numerous and if their mother cannot otherwise get them off her hands, she must send them abroad into the world. » (Punch , 1850)

\section{Introduction}

1 Le $\mathrm{XIX}^{\mathrm{e}}$ et les premières décennies $\mathrm{du} \mathrm{xx}^{\mathrm{e}}$ siècle sont marqués, en Europe, par d'importants mouvements migratoires, encouragés d'une part par les appels des territoires "neufs", colonies ou ex-colonies, pour lesquels l'accroissement de la population est essentiel au peuplement et au développement économique, d'autre part par une poussée démographique sans précédent (Courville, 2003 : 9). Les régions du nordouest de l'Europe, des îles Britanniques à la vallée du Rhin, sont les plus touchées. La population anglaise et galloise passe ainsi de 8,8 millions d'habitants en 1801 à 17,9 millions en 1851 puis 22,7 millions en 1871 , tandis que la révolution industrielle provoque un exode des campagnes vers les villes qui, à partir du milieu du siècle, abritent désormais la majorité de la population ${ }^{1}$. C'est dans ce contexte que le recours à 
l'émigration comme solution aux difficultés posées de part et d'autre se répand. La Grande-Bretagne, qui connaît alors son apogée comme puissance coloniale ${ }^{2}$, est, de fait, la région d'Europe qui contribue le plus à ces vagues migratoires ${ }^{3}$, principalement à destination de l'Australie, de la Nouvelle-Zélande, du Canada et des États-Unis, autant de régions où le besoin de main-d'œuvre est criant.

2 Si cette émigration est, au début du siècle, principalement masculine, la proportion de femmes ne va par ailleurs cesser de croître, encouragée et assistée par un certain nombre d'initiatives privées et publiques. Il ne s'agit pas encore, à ce stade, de servir un objectif ouvertement impérialiste, mais plutôt de répondre aux besoins des colonies tout en résolvant certains problèmes domestiques liés à la pauvreté ainsi que, de plus en plus, à l'excédent de femmes par rapport aux hommes révélé par les recensements successifs ${ }^{4}$.

3 Si cette question de l'émigration féminine de la Grande-Bretagne vers ses colonies a, ces vingt dernières années, suscité un intérêt croissant parmi les chercheurs, c'est la période ultérieure aux années 1860 qui a donné lieu à la littérature la plus abondante ${ }^{5}$. Aussi cet article se propose-t-il de se pencher plus particulièrement sur la vingtaine d'années précédente afin d'examiner, notamment, les motivations et les discours ayant sous-tendu les premières initiatives. Nous verrons ainsi que l'évolution dans la façon de percevoir cette émigration, envisagée dans un premier temps comme solution à la pauvreté puis comme remède au déséquilibre démographique dont semble souffrir le pays, s'accompagne d'un déplacement de la catégorie d'émigrantes visées de la classe ouvrière à la classe moyenne et donne lieu, dès lors, à deux types de discours que l'on pourrait aujourd'hui qualifier respectivement - quoi de de façon réductrice - de féministe et d'antiféministe. Avançant, pour un même projet, deux objectifs différents, basés sur deux conceptions a priori opposées de la femme - l'une relevant de la sphère publique, l'autre de la sphère privée -, ces discours sont néanmoins marqués par de nombreuses hésitations qu'il convient de relever et qui sont en partie attribuables à des tiraillements entre pragmatisme et idéologie.

\section{Le Fund for Promoting Female Emigration}

4 L'émigration vers ses colonies et dominions a souvent été un moyen pour la GrandeBretagne de se débarrasser d'une population encombrante. Celle-ci s'est tout d'abord faite vers les États-Unis puis, à partir des années 1820 (à l'exclusion des forçats), l'Australie et la Nouvelle-Zélande et, enfin, au début des années 1830, le Canada. C'est à cette période, en particulier, que, face à l'augmentation de la pauvreté et aux désordres que font craindre les difficultés cycliques de l'économie, le gouvernement britannique commence à s'y intéresser comme moyen de peupler l'Empire de citoyens britanniques tout en soulageant la misère en Grande-Bretagne. De fait, vers la fin des années 1840, l'émigration est plus que jamais considérée comme la solution par excellence aux maux dont souffre alors le pays, qu'il s'agisse de la surpopulation urbaine, de la famine qui touche l'Irlande, ou encore des clearances des Hautes Terres écossaises. C'est dans ce contexte que la Colonial Land and Emigration Commission (CLEC), fondée en janvier 1840, se voit confier la responsabilité de la gestion des terres dans les colonies britanniques et utilise les bénéfices des ventes pour promouvoir et réguler l'émigration vers ces mêmes colonies. Elle sélectionne les émigrants (jeunes servantes célibataires, jeunes couples avec ou sans enfants, ouvriers agricoles, bergers ou artisans qualifiés) en fonction de leur contribution potentielle au développement du territoire et leur fournit les informations nécessaires 
sur les destinations et les moyens de transport (Tran, $2010: 121$ ). Ils sont plus de 339000 à bénéficier de son aide entre 1847 et 1869, chiffre qui, pour l'ensemble du XIX ${ }^{e}$ siècle, va s'élever à près de 745000 sur un total de 1,5 millions d'émigrants britanniques (Snow, $1931: 247)$.

5 Si cette émigration est principalement ouvrière et essentiellement masculine, on note, au fil des années, une augmentation régulière du nombre de migrantes. Ainsi, tandis qu'en 1820 , elles ne représentent que $20 \%$ des partants vers les États-Unis, leur proportion atteint $35 \%$ en 1831 et $39 \%$ en 1841. Elles voyagent alors majoritairement en famille, en tant qu'épouse, fille ou domestique (seules $16 \%$ voyagent seules [Courville, 2003 : 33]). À l'exception de quelques rares initiatives de la part du gouvernement visant à encourager et encadrer l'émigration d'orphelines vers l'Australie (Molinari, $2018: 18$ ), l'émigration organisée et assistée de femmes célibataires ne s'organise quant à elle qu'à partir de 1849 , cette fois à l'initiative de philanthropes. La première organisation d'envergure de ce type est le Fund for Promoting Female Emigration, créée en décembre 1849 par deux députés Tory, Lord Herbert et Lord Ashley, afin de venir en aide aux modistes et couturières en leur procurant l'assistance logistique et matérielle nécessaire pour émigrer vers les colonies. L'intérêt porté à ces dernières a commencé quelques années plus tôt, suite à la publication, en 1843, d'un rapport de la Commission sur le travail des enfants. La description des conditions de vie et de travail de quelque quinze mille jeunes femmes issues des classes moyennes inférieures et forcées de travailler jusqu'à 18 heures par jour dans les mansardes et arrière-boutiques londoniennes pour un salaire de misère au point de mourir prématurément ou, pire, d'avoir recours à la prostitution pour survivre, n'a pas manqué de heurter les sensibilités (House of Commons, $1843: 555)^{6}$. Les " couturières en détresse ", ainsi qu'on les appelle, deviennent pendant quelques années une cause célèbre, dépeintes au travers de romans, nouvelles et poésie, et défendues dans la presse ${ }^{7}$. Au mois de novembre 1849, le journaliste Henry Mayhew publie une série d'articles dans le Morning Chronicle dans lesquels il dénonce de nouveau les conditions abjectes d'exploitation des ouvrières londoniennes et le recours inévitable à la prostitution de ces dernières et appelle les classes aisées à agir. C'est en réponse à cet appel que, le 5 décembre, Sidney Herbert annonce, dans les pages de ce même journal, sa décision de créer un fonds afin de venir en aide à ces jeunes femmes en leur permettant d'émigrer. L'objectif est clairement annoncé : il s'agit non seulement de les sauver de la pauvreté mais aussi, et surtout, de les empêcher de tomber dans la prostitution :

33,500 women engaged in this one trade, of whom 28,500 are under 20 years of age, and of these a large portion living, or attempting to live, on sums varying from $4 \frac{1}{2} d$. to $2 \frac{1}{2} d$. a day! [...] We should offer to those who still have health and years before them and above all who still have character, the means of escape from a country where their only possible calling brings them ruin, to a land which offers them the prospect of a home where they may dwell in comfort and honour. (Herbet, $1849: 4$ )

6 Herbert parvient à réunir autour de lui un certain nombre de dignitaires, parmi lesquels Lord John Russell, le marquis de Westminster, le conte de Harrowby, ainsi que les Rothschild et plusieurs membres de l'Église anglicane. On compte par ailleurs, en tête des souscripteurs, la reine Victoria et le prince Albert. Tous reçoivent l'assurance que «ne seront sélectionnées pour émigrer que les femmes de bonne réputation. Elles ne doivent pas porter le moindre soupçon de tâche ou de déshonneur qui pourrait ruiner leur avenir à leur arrivée dans leur nouveau pays ${ }^{8}$ ". L'initiative fait rapidement parler d'elle: en janvier 1850, le Penny Illustrated News, après avoir cité Mayhew et fourni des statistiques sur l'excédent de femmes en Grande-Bretagne et sur leur pénurie en Australie, conclue : 
"For those of them who have not made their misery perpetual by falling into sin there is hope. Mr Sydney [sic] Herbert has attracted the attention of the public to the most natural means of relief by recommending emigration " (Alexander, 2003 : 111); et Punch de confirmer, le même mois, dans un article intitulé « Our Female Supernumeraries » :

It is lamentable that thousands of poor girls should starve here upon slops, working for slopsellers, and only dying old maids because dying young, when stalwart mates and solid meals might be found for all in Australia. Doubtless, they would fly as fast as the Swedish hen-chaffinches-if only they had the means of flying. It remains with the Government and the country to find them wings. (1850:13)

Si l'émigration de ces jeunes femmes suscite autant d'intérêt et de soutien, ce n'est donc pas uniquement en raison de leur condition, mais également, comme l'illustrent ces deux articles, en raison du déséquilibre démographique révélé par le recensement de 1841, à savoir un excédent de plus de 300000 femmes sur une population de 15,9 millions en Angleterre et au pays de Galles. En envoyant de jeunes coutières en Australie et au Canada, le Fund for Promoting Female Emigration se propose de fait de faire d'une pierre deux coups, en résolvant à la fois les problèmes liés à cet excédent de femmes en métropole et ceux liés à leur pénurie dans les colonies. Dans ces premiers discours, toutefois, à la différence de ceux qui suivront une vingtaine d'années plus tard, il convient de noter que cet excédent n'est pas présenté comme nuisible d'un point de vue social, mais d'un point de vue économique, dans la mesure où il conduit à une dévalorisation des salaires et, de fait, à une hausse de la pauvreté.

Any women so emigrating is a woman saved; and further, if any numbers go out, the status of the remainder is bettered. The competition is diminished, and wages pro tanto improved. (Herbert, 1849:4)

Throughout Great Britain the male population is greatly outnumbered by the female. By the census of 1841 it appeared that there were 320,000 more women than men, and so great has been the amount of male emigration during the last nine years, that the excess of females may be fairly supposed to have reached half a million. In the British colonies, on the other hand, the inequality is reversed; in 1847 there were in New South Wales only 41,000 females to 83,000 males; in South Australia only 13,000 females to 17,000 males. Similar disproportion exists in Van Diemen's Land and other colonial dependencies. It is proposed to take measures with a view to abate this double evil; - to supply the colonies with the population which is the crying want of society there, and at the same time to redress this increasing grievance of the mother country, to rescue from penury and misery those who emigrate, and by diminishing the unnatural excess of female labour, to better the condition of those who remain. (The New Zealander, 29 mai $1850: 2$ )

8 On peut en effet espérer, en réduisant le nombre d'ouvrières travaillant dans la confection, faire augmenter les salaires de celles restées au pays. De fait, Herbert attribue les causes de la misère dans laquelle se trouvent ces milliers de femmes à la concurrence grandissante entre «capitalistes » et, dans un contexte d'explosion démographique, à la nécessité, pour les travailleurs, d'entrer en concurrence les uns avec les autres et à accepter des salaires toujours plus bas pour espérer obtenir du travail. Cette concurrence est d'autant plus sévère dans la confection, explique-t-il, dans la mesure où celle-ci est l'un des seuls secteurs ouverts aux femmes en termes d'opportunités d'emploi :

Their choice is very limited, and as their field of employment is narrower, so is it, proportionably, far more crowded. This needle-working, which is one of the largest, is the most over-crowded of all trades; in none, consequently, has the reduction in the price of labour and the cost of the article produced been so great. (Herbert, 1849:4) 
Parce qu'il semble inenvisageable, dans le contexte de montée du libéralisme, de légiférer afin de réglementer les prix ou les salaires, la solution est donc de jouer sur l'offre et la demande.

9 Malgré l'absence de soutien de la part des commissaires à l'émigration du CLEC, qui estiment que les colonies ont avant tout besoin de filles de ferme et de domestiques, les voyages se succèdent vers le Canada et l'Australie. La presse britannique ne tarit pas d'éloges au sujet de la société, et compare favorablement l'efficacité de cette dernière à celle du gouvernement. Un foyer est créé à Hatton Garden, à Londres, afin d'héberger, pendant quelques semaines avant le départ, les candidates au voyage. Quelque quarante jeunes femmes y sont recueillies et nourries, et reçoivent une formation de domestique qui leur permettra de trouver plus facilement un emploi.

D'autres initiatives voient le jour à la même période à travers le pays : durant l'été 1849, la National Benevolent Emigration Society entreprend d'aider les orphelines de militaires et de membres de professions libérales à émigrer (si le département de l'émigration au sein du Colonial office y est favorable, le projet est néanmoins abandonné suite aux réactions virulentes des colonies, qui disent avoir besoin d'ouvrières et non de jeunes filles de bonne famille). En Écosse, le Shetland Female Emigration Fund fournit l'équipement nécessaire au voyage à des femmes et jeunes filles écossaises dont le voyage est financé par le gouvernement. La British Ladies Female Emigration Society, enfin, fondée en 1849 par Lady Mary Jane Kinnaird, si elle ne vise pas à promouvoir l'émigration féminine, œuvre pour sa part à améliorer les conditions de traversée en procurant des «matrones » aux navires qui transportent des femmes seules vers l'Australie et la Nouvelle-Zélande et, plus tard, le Canada.

\section{Préoccupations matrimoniales et classe sociale}

11 Si l'émigration féminine est présentée par ses défenseurs avant tout comme un moyen d'aider les couturières à échapper à la pauvreté, la question devient rapidement associée dans la presse à celle des opportunités matrimoniales qui s'offriront, une fois dans les colonies, aux femmes célibataires qui tenteront l'aventure (Punch, 1850 : 14). Le lien établi entre les deux s'accroît encore suite à la publication des résultats du recensement de 1851 qui incluent, pour la première fois, des données relatives au statut marital de la population et révèlent, outre un excédent, cette fois, de 500000 femmes par rapport à la population masculine $e^{9}$ l'existence de deux millions et demi de femmes célibataires sur une population de vingt millions pour l'Angleterre et le pays de Galles (Levitan, 2008 : 363). Les chiffres et les commentaires qui accompagnent le recensement sont largement relayés par la presse et confirment alors ce qui n'avait été jusqu'à présent qu'un ressenti, transformant une préoccupation mentionnée occasionnellement par certains hommes politiques et écrivains en un véritable problème social. La question de l'émigration féminine est dès lors clairement redéfinie en de nouveaux termes: celle-ci ne doit plus seulement être un moyen d'aider des femmes pauvres à échapper à la pauvreté, mais également de trouver un mari aux célibataires.

12 Cette rhétorique du mariage, au moment où elle se répand dans la presse (Household Words , janvier 1852: 414 ; The Times, janvier 1852: 4), reste très peu employée par le Fund for Promoting Female Emigration lui-même. En 1852, ce dernier met au contraire en garde ses protégées contre des mariages hâtifs conclus dès leur arrivée dans la colonie et conseille à 
celles-ci de s'installer et de devenir financièrement autonome avant de trouver, «en temps voulu, des hommes bons et dignes qui les épouseront et leur assureront un avenir heureux " (The Times, août 1852: 8). Si ces émigrantes doivent, à terme, devenir les épouses de colons, il devient impossible d'ignorer encore longtemps la question du mariage et celle, connexe, des catégories sociales dont celles-ci devront être issues. Alors que Household Words, l'hebdomadaire de Charles Dickens, encourage ses lecteurs à souscrire au Fonds de Sidney Herbert (Hartley, 2009: 1999), la société doit au même moment affronter les critiques de la presse de part de certaines colonies: malgré les efforts faits pour sélectionner les candidates, certaines émigrantes ne sont pas les " couturières en détresse » et "sans tâche » que l'on s'est donné pour objectif d'aider mais, dénonce-t-on, des jeunes femmes de petite vertu parties chercher un climat plus clément et les premiers chercheurs d'or. Parce que le lien, de fait, est désormais souvent fait entre les couturières et la prostitution, la société préfère, à partir de 1853, recruter parmi les femmes des catégories sociales plus élevées (Hammerton, 1979 : 206), évolution qui reflète en grande partie celle des attitudes et du discours sur l'émigration en général. L'émigration devient en effet à la même période plus respectable et, avec la ruée vers l'or en Australie, à partir de 1852, cesse d'être associée à la pauvreté et la criminalité - ce sont principalement des hommes de la classe moyenne qui partent chercher fortune. Le travail de Sidney Herbert, quoique limité dans le temps (la société cesse ses activités en 1853 suite à des difficultés financières, après avoir envoyé plus de mille jeunes femmes vers l'Australie, l'Amérique du Nord, la Nouvelle-Zélande, le Canada et le cap de Bonne Espérance) contribue à cet égard à produire un climat plus favorable à l'émigration des femmes des classes moyennes même si, et le problème va devenir un sujet de discorde de plus en plus évident au fil du temps, le profil de ces dernières ne correspond pas, là non plus, aux demandes essentiellement domestiques des colonies, qui vont accuser la métropole de les utiliser comme réceptacle de « femmes inutiles ».

Le fait est que cette préoccupation pour l'origine sociale des jeunes femmes concernées doit moins à l'intérêt des colonies qu'au fait que le célibat apparaît principalement problématique pour les femmes de la classe moyenne. Les jeunes femmes de la classe ouvrière, estime-t-on, trouveront toujours trouver un emploi dans les usines de filature et de confection ou dans la domesticité. Celles de la classe moyenne ont, en revanche, peu d'opportunités d'emploi qui s'offrent à elles et, outre la menace de la pauvreté à laquelle elles sont confrontées, posent, selon certains, une menace à la stabilité de la société victorienne en ne remplissant pas le rôle qui devrait être le leur : se marier et enfanter ${ }^{10}$. La chute du taux de natalité que l'on observe à partir des années 1850 au sein des classes moyennes commence par ailleurs à susciter quelques inquiétudes quant à l'avenir de la « race» anglo-saxonne. Envoyer les femmes célibataires vers les colonies promettrait donc non seulement de libérer la métropole de leur présence mais également de contribuer à la reproduction de cette dernière dans d'autres territoires de l'Empire.

\section{Entre motivations « féministes » et « antiféministes »}

14 La disparition du Fund for Promoting Female Emigration, en 1853, ainsi que le début de la guerre de Crimée la même année vont faire oublier pendant quelques années ces préoccupations. Quand elles resurgissent, au début des années 1860, suite à la publication des nouveaux chiffres du recensement, le nombre croissant de femmes célibataires que 
ces derniers font apparaître fait toutefois prendre au débat une nouvelle ampleur. Pour certains, la présence dans le pays d'un nombre aussi important de femmes aux «existences manquées» (en français dans le texte), notamment au sein de la classe moyenne, est «malsaine ", «l'un des symptômes les plus menaçants de quelque chose de gravement et radicalement mauvais » (Greg, 1862: 5, 6, 30). La solution présentée par William Rathbone Greg dans un article de la National Review intitulé «Why Are Women Redundant? » n'est donc surtout pas d'améliorer les opportunités d'emploi et de salaires de celles qu'il taxe de « superflues » et d'« inutiles » dans la mesure où cela risquerait de les installer dans cette situation, mais bien de se débarrasser d'elles en les envoyant vers les colonies, où le déséquilibre démographique est inverse :

There are hundreds of thousands of women [...] scattered through all ranks, but proportionally most numerous in the middle and upper classes, - who have to earn their own living, instead of spending and husbanding the earnings of men; who, not having the natural duties and labours of wives and mothers, have to care out artificial and painfullysought occupations for themselves; who, in place of completing, sweetening, and embellishing the existence of others, are compelled to lead an independent and incomplete existence of their own. (Greg, 1862:5)

Cet article, qui suscite de nombreuses réactions, d'approbation plus que de condamnation d'ailleurs, illustre parfaitement le changement qui, parallèlement à la transformation de la question de l'excédent de femmes d'un problème anecdotique en une inquiétude nationale appuyée par des statistiques, est en train de s'opérer en matière de discours et de politique d'émigration féminine : tandis que les partisans de l'émigration des femmes célibataires ont jusqu'à présent mis l'accent sur les difficultés rencontrées par ces dernières en Grande-Bretagne et sur les opportunités que pourrait leur offrir une nouvelle vie dans les colonies, certains, comme Greg, n'hésitent désormais plus à mettre en avant la nécessité, pour le pays, de se débarrasser de ces femmes encombrantes. L'émigration féminine n'est plus tant, pour ceux-ci, un service à rendre aux femmes qu'un service à rendre au pays.

Parce que le débat s'inscrit dans un contexte qui est également celui de l'émergence d'un mouvement féministe organisé, il n'est pas surprenant que pour d'autres, proches du groupe de Langham Place ${ }^{11}$, le problème représenté par l'excédent de femmes doive plutôt être posé en termes d'absence d'opportunités d'emploi pour les femmes des classes moyennes qu'en termes de chiffres. L'émigration féminine, par conséquent, si elle est tout à fait acceptable, voire recommandable, dans la mesure où elle peut permettre à des femmes qui ne parviennent pas à trouver un emploi en Grande-Bretagne de tenter leur chance ailleurs, doit uniquement être envisagée comme une solution temporaire en attendant une amélioration des conditions en métropole. De fait, la publication de l'article de W.R. Greg coïncide, à un mois près, avec la création d'une nouvelle organisation liée à la Société pour la promotion de l'emploi des femmes (Society for Promoting the Employment of Women ${ }^{12}$ ) : la Female Middle Class Emigration Society (FMCES). Formée en mai 1862 à l'initiative de Maria Rye, et avec l'assistance d'autres membres fondateurs du groupe de Langham Place, la société, présidée par Lord Shaftsbury, vise à venir en aide aux femmes célibataires de la classe moyenne qui ne bénéficient pas des financements du gouvernement auquel sont alors éligibles les femmes de la classe ouvrière. Outre une assistance financière procurée sous la forme de prêts sans intérêts financés par des dons privés, la société établit des contacts, au départ et à l'arrivée, et aide des jeunes femmes instruites à trouver un emploi en Australie, Nouvelle-Zélande, Afrique du Sud et au Canada. Les demandes pleuvent, selon les dires de l'association qui, 
la première année, aide officiellement 54 femmes, et officieusement 315, à partir (FMCES Annual Report, $1861:$ 7-8, dans Perry, $2001: 151$ ).

Deux catégories sont visées par cette initiative: d'une part les gouvernantes; d'autre part, un groupe hétérogène de jeunes femmes issues des classes moyennes les plus modestes, plus instruites que les générations précédentes et ne se destinant pas à la domesticité en Angleterre ${ }^{13}$. Parce que travailler de ses mains est, selon Maria Rye, préférable à la pauvreté, ces dernières doivent toutefois être prêtes à accepter dans les colonies des emplois "supérieurs » de domestiques (bien que la notion reste floue) et avoir, si possible, un minimum de compétences en cuisine, lessive ou couture ${ }^{14}$. Cette vision de l'émigration comme moyen d'améliorer la condition des femmes britanniques signifie que l'initiative est rapidement soutenue par l'English Woman's Journal ${ }^{15}$ qui, pendant plusieurs années, va régulièrement publier lettres d'immigrantes et articles présentant les avantages et inconvénients de l'émigration avec, comme message dominant, l'idée que « ces filles en surnombre que l'on néglige trouveront un foyer et le bonheur dans le Nouveau-Monde et [que] ce qui est en apparence un fléau deviendra une véritable bénédiction ${ }^{16} »$.

Si Maria Rye ne peut, elle-même, être qualifiée de féministe (elle quittera d'ailleurs la SPEW lorsque celle-ci commencera à faire campagne pour le suffrage féminin), son travail s'inscrit donc bien dans les actions du mouvement féministe naissant. Or, la coexistence de deux types de discours au sujet de l'émigration féminine est problématique, et le lien qui ne manque pas d'être fait avec le discours de Rathbone Greg fait que certaines militantes sont de plus en plus réticentes à s'associer à une telle initiative. C'est le cas de Frances Power Cobbe qui, dans un article intitulé «What Shall We Do with Our Old Maids? », compare l'émigration féminine assistée à l'expulsion de bagnards vers les colonies. La société britannique, explique-t-elle, tant qu'elle refusera aux femmes l'accès à certains emplois respectables, condamnera les femmes seules à l'unique choix entre l'« exil et la faim» (Cobbe, 1862 : 599). La réédition de l'article de Greg en 1868 et 1869 provoquera de semblables réactions de la part des féministes concernant le sort de celles que l'on ira désormais jusqu'à taxer de "superflues ». Jessie Boucherett, dans un essai intitulé " How to Provide for Superfluous Women ", expliquera ainsi de nouveau que le problème n'est pas une question de chiffres mais d'offre et de demande pour certaines catégories de population et que la solution n'est donc pas de déplacer les femmes seules d'un endroit à un autre, mais de les rendre plus utiles (1869 : 31). Mary Taylor, qui a ellemême émigré seule en 1845 en Nouvelle-Zélande suite à la mort de son père, répondra quant à elle à Greg :

The men who emigrate without wives, do so because in their opinion they cannot afford to marry. The curious idea that the women, whom they would not ask in England should run after them to persuade them would be laughable it is were not mischievous. Those who adopt it must dispense with that cultivated forethought that makes both sides wish for some provision for the future before entering into matrimony. (1870:43)

Il serait toutefois faux de croire que les propos de Rathbone Greg choquent celles qui sont, au début des années 1860 , le plus étroitement liées aux projets d'émigration. Certains des articles ou des lettres reproduits dans l'English Woman's Journal tiennent en fait un discours très similaire au sien, que l'on qualifierait pourtant aisément aujourd'hui d'" anti-féministe ». Ainsi, dans un article publié en octobre 1862, «A Lady Who Has Resided Eleven Years in One of the Australian Colonies ", l'auteur reprend les propos de Greg et notamment l'idée que les femmes seules sont nuisibles à la société : 
The question how to balance the account, how to transfer [women] from the place where they are not wanted, and where they may even become injurious, to the place where they will be valued as they deserve, and benefit instead of injuring those around them. ("Female Emigration... », The English Woman's Journal, 1862 : 73)

Il est par ailleurs évident, à la lecture de ces lettres, que certaines émigrantes ellesmêmes se considèrent comme appartenant à un groupe problématique : «J'aime toujours autant [l'Angleterre] » écrit l'une d'entre elles, «mais je peux gagner plus d'argent ici et toujours trouver, je pense, quelque chose à faire; nous sommes suffisamment nombreuses chez nous ${ }^{17} »$.

\section{Les sociétés d'émigration féminine : bureaux de placement ou agences matrimoniales?}

21 Outre une possible association avec les propos de William Rathbone Greg, la FMCES se trouve rapidement confrontée à un autre problème : suite à la conférence donnée par Maria Rye à l'Association for the Promotion of Social Science en avril 1862, au cours de laquelle le projet a été lancé, s'est en effet établi un échange dans les pages du Times quant à l'objectif réel de la société. En insinuant, dans une lettre au rédacteur en chef, que la FMCES n'est rien d'autre qu'une agence matrimoniale, l'écrivain et réformateur social Charles Kingsley, bien que soutenant le projet, a en effet démarré une controverse sur les mérites de la "colonisation matrimoniale» (The Times, 11 et 28 avril 1862). Maria Rye réfute ces propos, soutenant que si les femmes étaient moins exigeantes dans le choix d'un mari en Grande-Bretagne, elles pourraient facilement en trouver un parmi leurs inférieurs et qu'il n'y a donc pas de raison de penser qu'elles seront moins regardantes une fois installées dans les colonies. L'objectif de l'organisation est bien, insiste-t-elle, d'aider ces jeunes femmes à trouver un emploi, le mariage restant, quant à lui, une affaire privée (The Times, 29 avril 1862).

Les activités de la FMCES sont toutefois de nouveau sujettes à critiques avec la publication, quelques mois plus tard, d'un article de la Saturday Review intitulé «The Export Wife Trade », dans lequel l'auteur s'en prend violemment à la société, qu'il accuse de vouloir utiliser les colonies comme déversoir ( outfall sewer ») de la Grande-Bretagne et qui associe les émigrantes aux catégories «à problème » tels que «les indigents, les pénitents ou les forçats ».

Stimulated emigration only carries those whom we are most eager to be rid of here [...] however pure may be the philanthropy of those who inaugurate schemes of emigration for the relief of over-stocked labour markets, it is always the least estimable portion of the superfluity who are selected for the operation. It is the most incorrigible convict, the most incapable pauper, the most unmarketable governess, the most ineligible maiden, who is selected for the employer or the husband of the new country [...]. The colonists are tired of becoming customers for the goods which can find no sale in England-chipped statuettes, spoiled engravings and old maids. (6 septembre $1862: 276$ )

En organisant ce que l'auteur qualifie de "demande en mariage collective », Maria Rye et les autres philanthropes britanniques impliqués dans le projet sont par ailleurs rendus coupables de froisser les sensibilités des colons en agissant comme si le mariage n'était pour ces derniers qu'un commerce et non une affaire de sentiments :

There is something prosaic, nay even brutal, in the unwincing candour of Miss Rye's hymeneal arrangements. The least romantic squatter, the most grovelling digger, will be revolted by seeing the ways of courtship made so straight and flat. The news that a cargo of 
hypothetical brides had arrived, and was waiting in the port town to be looked at, would scare away every possible bridegroom from coming anywhere near the coast. néo-zélandaise ${ }^{18}$ n'est pas le seul du genre et les réactions dont se font le relais, durant les mois de l'été 1862, les journaux de la colonie de Victoria, en Australie, sont nombreuses. Une lettre adressée au Melbourne Argus en juin 1862 traduit ainsi le sentiment de certains colons à l'idée de se voir imposer des jeunes femmes que l'auteur compare à des articles potentiellement « invendables » :

We may bring the young women here, but what if they do not suit the young men? What shall we do with the articles which don't "move off", and the goods which are found unsaleable? The disproportion which at present exists between the sexes in this colony is undoubtedly a great social evil, but we doubt if it is to be cured by the indiscriminate importation of educated young women from England. Our bachelors will probably insist upon their right of free selection. They may not want to be married at all, however odious this determination may appear to Miss Rye and her friends. Moreover, is there nothing due to existing interests? Are our spinsters already on hand to be neglected in favour of the imported article? It is a fallacy to suppose that our home market is so entirely bare as that we are compelled to depend upon foreign produce. (Melbourne Argus, 17 juin 1862 : 4)

Face aux attaques dont elle est victime, la FMCES se trouve forcée de publier un démenti dans l'English Woman's Journal, expliquant qu'elle n'a jamais, dans ses propos ou ses actions, fait miroiter à ses émigrantes l'idée d'un mariage à l'arrivée :

The essential feature in the Society's plan is not to attempts to relieve the mother-country by throwing upon the colonies heterogeneous masses of women [...] it would be a most serious error to regard their labours as in any way intended to encourage the idea, revolting to every feminine instinct, of seeking marriage as a gambler seeks a prize in a lottery. Miss Rye and her coadjutors distinctly and emphatically declare, that they have never, by word or deed, held out to any woman, the prospect of marriage as an inducement to emigrate... If suitable marriage comes in an emigrant's way, let her accept it and find honour and happiness and opportunities of usefulness in it. But a true woman does not cease to be such when she crosses the seas. ("Female Emigration... », $1862: 83$ )

L'émigration féminine, souligne l'auteur de l'article, n'est qu'un moyen parmi d'autres d'améliorer la condition des femmes, mais la meilleure façon de venir en aide à ces dernières reste la suppression des obstacles au travail féminin qui existent en GrandeBretagne. Ces démentis sont vains. Les attaques continuent et, lorsque le Conway arrive à Rockhampton (Queensland, Australie) en décembre 1862, la nouvelle selon laquelle se trouvent à son bord 141 célibataires, dont 96 gouvernantes sélectionnées par Miss Rye, suscite de nouvelles réactions au sein de la communauté locale et la publication, dans le Brisbane Courier, de "The Wife Export Trade ", l'article de la Saturday Review publié trois mois auparavant.

L'une des raisons à la confusion qui entoure les objectifs de la FMCES est sans aucun doute le fait que, deux mois à peine avant sa création et la publication de l'article de W. R. Greg dans la National Review, une autre organisation a été créée par une philanthrope anglicane, Angela Burdett Coutts, avec pour objectif affiché de procurer des épouses aux colons et aux mineurs d'or de la Colombie-Britannique. Le projet a été lancé en réponse à un courrier adressé par le révérend R. C. Lundin Brown, pasteur de la ville de Lillooet, à l'évêque d'Oxford en 1861 et portant à l'attention de ce dernier le problème du manque criant de femmes dans la colonie où des d'hommes de tous âges sont venus faire fortune dans les mines d'or :

Dozens of men have told me they would gladly marry if they could. I was speaking one evening on the subject of the dearth of females and mentioned my intention of writing to beg 
that a plan of emigration may be set on foot; whereupon one member of the company immediately exclaimed, "Then Sir, I pre-empt a wife", and another and another, all round the circle of those listening to me earnestly exclaimed the same. Fancy the idea of preempting a wife! Yet, I assure you this touches the root of the greatest blessing which can now be conferred upon this colony from home. (Peake, $1959: 40$ )

L'objectif premier de la Columbia Emigration Society (CES), n'est pas, contrairement à la FMCES, de trouver du travail à des femmes sans emploi, mais bel et bien cette fois-ci de fournir des fiancées britanniques à des colons souhaitant "préempter» une épouse et, ce faisant, contribuer à protéger la nouvelle colonie (le territoire est devenu britannique en 1858) de la double menace de l'immoralité que représentent les relations sexuelles hors mariage et les alliances interraciales avec la population indigène (Perry, 2001 : 152). La société ne s'intéresse d'ailleurs pas aux célibataires de la classe moyenne qui causent tellement de tracas à W. R. Greg, mais va au contraire chercher ses candidates au départ dans les workhouses et les orphelinats où, en les prenant très jeunes, elle espère les sauver de la prostitution. Si, en Angleterre, les objectifs de la société ne sont pas exprimés de façon aussi directe - il s'agit avant tout, dit-on, d'encourager l'émigration de femmes « respectables et travailleuses » comme domestiques « comme première étape avant de procurer des épouses aux mineurs et aux colons »-, le surnom qui est rapidement donné en Colombie-Britannique au navire qui effectue la traversée en juin 1862 - le «bride ship »- ne laisse aucun doute quant à la façon dont son chargement est perçu. Après une traversée de trois mois, et malgré des tentatives pour tenir le lieu de leur arrivée secret, une soixantaine de jeunes filles sont accueillies par quelque trois cents hommes rassemblés à James Bay, Victoria.

[...] we went aboard the steamer yesterday morning and had a good look at the lady passengers, rapporte à cette occasion le Daily British Colonist. They are mostly cleanly, well-built, pretty looking young women [...]. Most appear to have been well raised and generally they seem a superior lot to the women usually met with on emigrant vessels. Taken altogether, we are highly pleased with the appearance of the "invoice" and believe that they will give a good account of themselves in whatever station of life they may be called to fill even if they marry lucky bachelor miners from Cariboo [...]. A large number of citizens visited Esquimalt yesterday and endeavoured to board the vessel, but were generally ordered off and returned from their fruitless errand with heavy hearts. (19 septembre $1862: 3$ )

Lorsque, six mois plus tard, le 12 janvier 1863, le Robert Lowe arrive à son tour à Victoria, ce sont cette fois près de mille hommes qui sont rassemblés sur les docks, s'attendant à voir débarquer, selon une fausse annonce faite trois mois plus tôt par le même journal, quelque cinq cents jeunes femmes célibataires. En l'occurrence, ce sont seulement trentesix jeunes filles originaires de Manchester, âgées de 12 à 19 ans et sans instruction (la majorité d'entre elles seront rapidement placées comme domestiques) (ibid.:176), envoyées par la Columbia Emigration Society qui débarquent à West Bay (Johnson, 2003 : 165-166).

Si les activités de la CES sont de courte durée - elles cessent après ces deux voyages (Kholi, $2003: 318$ ) -, elles suffisent à flouter les distinctions entre les deux sociétés et leurs discours respectifs auprès du grand public. Le fait que la CES et la FMES aient été, durant cette période, toutes deux gérées par des membres de l'Église anglicane et que leurs organisatrices aient partagé les mêmes convictions vis-à-vis de l'émigration féminine a par ailleurs abouti à des collaborations lors des deux traversées. Or, malgré des réticences vis-à-vis du discours et du manque de sélectivité dans le recrutement des candidates au départ de la CES, Maria Rye a non seulement accepté de superviser la traversée de juin 1862, mais n'a eu d'autre choix à cette occasion que de se plier aux 
attentes de la colonie et de n'envoyer, non pas les gouvernantes visées par la FMCES mais quarante jeunes femmes principalement issues de la classe ouvrière (Perry, 2001 : 152). Les réticences locales aux objectifs féministes de la FMCES telles qu'exprimées par le Daily British Colonist ne pouvaient être, il faut dire, plus explicites :

The English papers tell us that there is a college for the purpose of promoting the emigration of educated women; and it appears that a lady named Miss Rye, affected with the cacoethes scribendi, is the head mistress of the college, or at least a leading graduate [...].

We never knew a man with matrimony in his eyes who expressed any affection for "bluestockings" in general, and are certain that [...] it will be a great fall for women of the governess class to be compelled through sheer hunger and want to sink to the grade of comparatively uneducated domestic servants [...]. The women we want in this and other colonies are women prepared to rough it as well as ourselves, women who, while acting as domestic servants, the class we particularly lack, will possess all the fair graces of womanhood and the virtues which will make them an ornament to their sex, at once model servants as well as model wives. (« Hope for Education Women », $1^{\mathrm{er}}$ juillet $1862: 4$ )

De fait, l'inadéquation entre l'offre et la demande signifie que la FMCES peut difficilement envoyer en grand nombre des femmes des classes moyennes dans les colonies, où les débouchés pour des gouvernantes sont plus que rares. Aussi, parce que ses motivations sont plus philanthropiques que féministes, et plus pragmatiques qu'idéologiques, Maria Rye préfère-t-elle orienter ses efforts vers les jeunes femmes de la classe ouvrière avant de quitter définitivement la FMES pour se consacrer exclusivement à ces dernières.

Le flou qui entoure le discours de la FMCES au début des années 1860 s'estompe toutefois avec ce départ et l'arrivée de Jane Lewin à la tête de la société en 1865. La décision de cette dernière de revenir aux objectifs premiers de la FMCES en restreignant le recrutement des candidates aux gouvernantes marque, de fait, une rupture nette, non seulement avec la tradition philanthropique représentée par sa prédécesseure et le Fund for Promoting Female Emigration de Sidney Herbert mais, surtout, avec la notion d'une sphère domestique qui serait naturellement celle de la femme - se démarquant de fait des associations possibles avec le discours de Greg et celui de la Columbia Emigration Society . L'émigration féminine, afin d'être cohérente avec des principes féministes, doit être un moyen d'offrir aux candidates au départ émancipation et indépendance financière, non pas un travail domestique qui, non seulement imposerait aux émigrantes un déclassement social, mais ne serait, qui plus est, considéré que comme un prélude, voire une préparation au mariage. Cette conception de l'émigration féminine et le rejet d'un type supérieur de domesticité signifient toutefois que le travail entrepris à partir de 1865 par la FMCES n'aura, quantitativement, que peu d'impact (quelque trois cent femmes seulement émigreront par son biais entre 1862 et 1885), même s'il reste significatif du point de vue des actions entreprises à l'époque par le mouvement féministe naissant.

Les sociétés d'aide à l'émigration féminine qui se multiplieront par la suite (la FMCES ellemême sera absorbée par la Colonial Emigration Society en 1886, peu de temps après le départ de Jane Lewin, en 1881 [Ruiz, 2017 : 9]) ne seront quant à elles plus liées à un projet philanthropique ou féministe, mais s'inscriront clairement dans une optique impérialiste (Bush, 1994 ; Chilton, 2013 ; Ruiz, 2017). Entre 1888 et 1914, ce sont 15636 femmes environ qui émigreront par le biais de ces organisations créées afin de les convaincre de participer à cette aventure, les aider dans leur démarche et les accompagner lors de leur traversée vers le Canada, la Nouvelle-Zélande, l'Australie ou l'Afrique du Sud (Ruiz, $2017: 4)$. 


\section{Conclusion}

Durant la vingtaine d'années qui marque les débuts de l'émigration assistée des femmes célibataires, on assiste donc, en Grande-Bretagne, à une succession, mais également parfois à une juxtaposition de projets et de discours, qu'il s'agisse de la catégorie sociale des femmes visées, des motivations de ses partisans - les uns considérant l'émigration comme un moyen de débarrasser le pays de ces femmes surnuméraires, les autres comme un moyen d'aider celles-ci à trouver une vie meilleure -, ou encore des moyens par lesquels ce dernier objectif pourra être atteint, à savoir le mariage ou l'emploi. Les repositionnements, hésitations et parfois même contradictions que l'on observe alors dans les discours sont bien évidemment à relier aux changements économiques et sociaux de la période ainsi qu'à certaines préoccupations coloniales; ils sont également, en grande partie, le reflet de la difficulté de concilier exigences pragmatiques et considérations idéologiques, de classe comme de genre, interdisant aux uns, d'imaginer une remise en question de l'idéologie de la séparation des sphères qui permettrait aux femmes de la classe moyenne un accès au monde du travail, aux autres un possible déclassement social.

\section{BIBLIOGRAPHIE}

\section{Sources primaires}

" Address of the Rev. B. Noel to the Emigrants on Board the Northumberland ", The Times, 3 août $1852,8$.

"A Rainy Day on the Euphrates ", Household Words, vol. IV, 24 janvier 1852, 414, <www.djo.org.uk/ household-words/volume-iv/page-414.html> (20 octobre 2017).

«Arrival of the Tynemouth », The Daily British Colonist, Victoria, vol. 8, 19 septembre 1862, 3, < http://ia601000.us.archive.org/11/items/dailycolonist18620919uvic/18620919.pdf> (15 mars 2017).

BUTLER Josephine (1869), Woman's Work and Woman's Culture: A Series of Essays, Londres : Macmillan. CoBBE Frances Power (1862), « What Shall We Do with Our Old Maids? », Fraser's Magazine, 66, 599.

«Efforts in England in Aid of Emigration », The New Zealander, vol. 5, n 430, 29 mai 1850, 2, < https://paperspast.natlib.govt.nz/newspapers/NZ18500529.2.5> (10 mars 2017).

«Female Emigration. British Ladies Female Emigrant Society », The New Zealander, vol. 8, $\mathrm{n}^{\circ} 616$, 10 mars $1852,3$.

«Female Emigration Impartially Considered », The Englishwoman's Journal, vol. X, octobre 1862, 73.

«Female Middle Class Emigration, Paper read at the meeting of the Association for the Promotion of Social Science, London, 1862 ", The Englishwoman's Journal, vol. X, septembre 1862, 20. 
GREG William Rathbone (1869), Why Are Women Redundant?, Londres : N. Trübner \& Co.

HERBERT Sidney (1849), « To the Editor of the Morning Chronicle », The Morning Chronicle, 5 décembre $1849,4$.

« Hope for Educated Women », The Daily British Colonist, Victoria, vol. 8, $1^{\mathrm{er}}$ juillet 1862, 4, <http:// ia601001.us.archive.org/20/items/dailycolonist18620701uvic/18620701.pdf> (15 mars 2017).

HousE OF COMmons (1843), Reports from Commissioners: Children's Employment, Trade and Manufactures, Sessional Papers XIV, 555.

House of Commons Debates, 28 mai 1850, vol. 111, c. 433-452.

Melbourne Argus, 17 juin 1862, 4, <http://trove.nla.gov.au/newspaper/article/5716541/205273> (15 juin 2017).

« Our Female Supernumeraries-In a Series of Views », Punch 18, 1850, 13.

TAYLOR Mary (1870), The First Duty of Women: A Series of Articles Reprinted from The Victoria Magazine 1865-70, Londres : Emily Faithful.

«The Departure of Miss Rye for the Colonies », The Englishwoman's Journal, décembre 1862, 261.

« The Export Wife-Trade », The Saturday Review, 6 septembre 1862, 276.

« The Needlewomen's Farewell », Punch, 12 janvier 1850, 14.

\section{Sources secondaires}

ALEXANDER Lynn Mae (2003), Women, Work, and Representation: Needlewomen in Victorian Art and Literature, Ohio : Ohio University Press.

BOYER George R. (2004), « The Evolution of Unemployment Relief in Great Britain », Journal of Interdisciplinary History, 34(3), 393-433.

BusH Julia (1994), « “The Right Sort of Woman”: Female Emigrators and Emigration to the British Empire, 1890-1910», Women's History Review, 3(3), 385-409.

CHILTon Lisa (2013), Agents of Empire: British Female Migration to Canada and Australia, 1860s-1930, Canada : University of Toronto Press.

COURVILLE Serge (2003), Immigration, colonisation et propagande, Québec : Éditions Multimondes. DIAMOND Marion (1999), Emigration and Empire: The Life of Maria S. Rye, New York : Garland Publishing.

ENGELS Friedrich (1960), La situation de la classe laborieuse en Angleterre (traduction et notes par Gilbert Badia et Jean Frédéric), Paris : Éditions sociales. (Ouvrage original publié en 1845 sous le titre Die Lage der arbeitenden Klasse in England.)

GothARD Janice (2001), Blue China: Single Female Migration to Colonial Australia, Melbourne : Melbourne University Publishing.

HAMMERTON James (1979), Emigrant Gentlewomen: Genteel Poverty and Female Emigration, 1830-1914, Londres : Croom Helm.

HAMMERTON James (2013), « Feminism and Female Emigration, 1861-1886 », M. Vicinus, A Widening Sphere: Changing Roles of Victorian Women [1977], Bloomington et Londres : Indiana University Press.

HARTLEY Jennifer (2009), Charles Dickens and the House of Fallen Women, Londres : Methuen. 
JEFFREYS Sheila (1997), The Spinster and Her Enemies: Feminism and Sexuality, 1880-1930, Melbourne : Spinifex Press.

JoHnson Peter (2003), Voyages of Hope, the Saga of the Bride-Ships, Victoria : Touchwood Editions. KoHLI Marjorie (2003), The Golden Bridge: Young Emigrants to Canada, 1833-1939, Toronto : Natural Heritage.

KRANIDIS Rita (1998), Imperial Objects: Essays on Victorian Women's Emigration and the Unauthorized Imperial Experience, Twayne Publishers.

KRANIDIs Rita (1999), The Victorian Spinster and Colonial Emigration: Contested Subjects, Palgrave Macmillan.

Levitan Kathrin (2008), « Redundancy, the "Surplus Woman" Problem, and the British Census, 1851-1861 », Women's History Review, 17(3), 359-376, <http:// dx.doi.org/10.1080/09612020801924449>.

LEVITAN Kathrin (2011), A Cultural History of the British Census: Envisioning the Multitude in the Nineteenth Century, Londres : Palgrave Macmillan.

Mitchell Brian R. (1988), British Historical Statistics, Cambridge : Cambridge University Press. MOLINARI Veronique (2018), «The Emigration of Irish Famine Orphan Girls to Australia: The Earl Grey Scheme », M. Ruiz (dir.), International Migrations in the Victorian Era, Leiden : Brill.

OLSON James S. \& SHADLE Robert (dir.) (1996), Historical Dictionary of the British Empire: A-J, Westport, CT : Greenwood Publishing.

PARsons Timothy H. (1999), The British Imperial Century, 1815-1914: A World History Perspective, Maryland : Rowman \& Littlefield.

PEAKE Frank A. (1959), The Anglican Church in British Columbia, Vancouver : Mitchell Press.

PERRY Adele (1997), « "Fair Ones of a Purer Caste": White Women and Colonialism in NineteenthCentury British Columbia », Feminist Studies, 23(3), 501-524.

PERRY Adele (2001), On the Edge of Empire: Gender, Race, and the Making of British Columbia, 1849-1871, Toronto : University of Toronto Press.

RuIz Marie (2017), British Female Emigration Societies and the New World, 1860-1914, Londres : Palgrave Macmillan.

SHEPPERSON Wilbur S. (1957), British Emigration to North America Projects and Opinions in the Early Victorian Period, Minneapolis : University of Minnesota Press.

SNOW C. E. (1931), « Emigration from Great Britain », W. F. Willcox (dir.), International Migrations, vol. II : Interpretations, NBER, 237-260, <www.nber.org/chapters/c5111.pdf> (18 juillet 2017).

SWAISLAND Cecilie (1993), Servants and Gentlewomen to the Golden Land: The Emigration of Single Women from Britain to Southern Africa, 1820-1939, Oxford : Berg.

TRAN Tri (2010), Les migrations assistées et forcées des Britanniques au XIX siècle, Paris : L'Harmattan.

\section{NOTES}

1. Le recensement de 1851 révèle que la moitié de la population britannique vit désormais en milieu urbain. 
2. Quelque $26000000 \mathrm{~km}^{2}$ de territoire et 400 millions de personnes ont été ajoutés à l'Empire au cours du siècle (Parsons, 1999 : 3).

3. Avec 11,4 millions d'émigrants de 1815 à 1930, l'Angleterre et le pays de Galles précèdent l'Italie (9,9), l'Irlande (7,3) et l'Autriche-Hongrie (5) (Courville, $2003: 14)$.

4. On dénombre ainsi 1057 femmes pour 1000 hommes en Angleterre et au pays de Galles en 1801, 1054 en 1811, 1036 en 1821, 1040 en 1831, 1046 en 1841, 1042 en 1851, 1053 en 1861, 1054 en 1871 (Mitchell, 1988 : 9-10).

5. Hormis l'ouvrage incontournable de James Hammerton, Emigrant Gentlewomen: Genteel Poverty and Female Emigration, 1830-1914 (1979), il faut attendre les années 1990 pour voir apparaitre de nouvelles études telles que, notamment: Dea Birkett, Spinsters Abroad: Victorian Lady Explorers (1989) ; Charlotte Macdonald, A Woman of Good Character: Single Women as Immigrant Settlers in Nineteenth-Century New Zealand (1990) ; Lisa Chilton, Agents of Empire: British Female Migration to Canada and Australia, 1860-1930 (2007) ; Julia Bush, « The Right Sort of Woman': Female Emigrators and Emigration to the British Empire, 1890-1910», Women's History Review, 3(3), 1994, 385-409 ; Rita Kranidis, The Victorian Spinster and Colonial Emigration: Contested Subject (1999) ; Marie Ruiz, British Female Emigration Societies and the New World, 1860-1914 (2017).

6. La situation est également dénoncée par Friedrich Engels dans La situation de la classe laborieuse en Angleterre, en 1845 : «Ces établissements occupent une foule de jeunes filles - en tout, paraît-il, quinze mille - qui habitent et mangent dans la maison même où elles travaillent; elles viennent pour la plupart de la campagne et sont ainsi complètement les esclaves de leurs patrons. Pendant la saison fashionable, qui dure environ quatre mois de l'année, la durée du travail, même dans les meilleurs établissements, atteint quinze heures par jour et quand le travail presse, 18 heures ; cependant dans la plupart des boutiques, on travaille pendant cette période sans que la durée de travail soit nettement fixée, si bien que les jeunes filles n'ont par jour, que six heures tout au plus, souvent seulement trois ou quatre, parfois même deux heures sur vingt-quatre pour dormir et se reposer, quand elles ne sont pas contraintes de travailler toute la nuit, ce qui n'est pas si rare que ça! La seule limite à leur travail, c'est l'incapacité physique absolue de tirer l'aiguille une minute de plus. Il est arrivé qu'une de ces créatures sans défense, reste neuf jours de suite sans se déshabiller et ne puisse se reposer que quelques instants, à l'occasion, sur un matelas où on lui servait à manger des mets découpés en menus morceaux, afin qu'elle puisse avaler la nourriture le plus rapidement possible. » (Engels, $1960: 170)$

7. En octobre 1843, un article du Times rapporte l'histoire d'une couturière qui a, en toute illégalité, mis en gage les vêtements qu'on lui avait donné à confectionner parce que ses enfants et elle-même étaient en train de mourir de faim. Deux mois plus tard, le journal provoque un nouveau scandale en rapportant la tentative de suicide (et la tentative de meurtre sur son enfant) d'une chemisière.

8. "In the selection of emigrants one condition must never be lost sight of. None but women of good character must be assisted to go. There must be no taint or discredit upon them to mar their prospects when they arrive at their new home. » (Hartley, 2009 : 199)

9. La tendance est commune à la plupart des pays européens et due, entre autres, aux guerres et à une émigration essentiellement masculine.

10. William Farr, l'épidémiologiste et statisticien, souligne ainsi dans le rapport qui accompagne les chiffres du recensement de 1851: «[...] on the conjugal state of the population, [the nation's] existence, increase and diffusion, as well as manners, character, happiness and freedom, intimately depend. » (The Census of Britain in 1851, p. 35, dans Levitan, $2011: 128$ )

11. Le groupe, composé de militantes de la classe moyenne, a été créé en 1857 par Barbara Bodichon et Elizabeth Rayner Parkes afin de faire campagne pour l'éducation, l'emploi, le droit à la propriété des femmes mariées et le suffrage féminin.

12. Elle-même a été créée deux ans auparavant par Jessie Boucherett et Adelaide Anne Procter afin de former les femmes aux emplois de bureau et les aider à devenir financièrement 
indépendantes. La SPEW est soutenue par la puissante National Association for the Promotion of the Social Sciences, groupe de pression pour la réforme sociale constitué de certaines des figures politiques importantes du moment.

13. "[...] the half-educated daughters of poor professional men, and including the children of subordinate Government officers, petty shopkeepers, postmen, policemen and artisans generally. " (« A paper read at the meeting of the Association for the Promotion of social Science ", Londres, 1862, p. 26.)

14. "Every applicant is examined as far as possible, with regard to her knowledge of cooking, baking, washing, needlework, and housework, and is required to assist in these departments of labour, should it be necessary. » (FMCES, First Report, 1861-1862, p. 3, dans Kohli, 2003 : 319)

15. Créé en 1858 par Barbara Leigh Smith Bodichon et Bessie Rayner Parkes.

16. «[...] superabundant and neglected daughters will find home and happiness in the New World, and the seeming curse will become a real blessing. » («Female Emigration... », English Woman's Journal, $1^{\mathrm{er}}$ octobre $1862: 80$ )

17. «I love [England] as ever, but I can earn more money here and I expect always find something to do, there are enough of us at home " (Levitan, 2011 : 138).

18. Empire (Sydney, NSW, 1850-1875), 18 novembre 1862; Otago Daily Times, $\mathrm{n}^{\circ} 300,5$ décembre 1862 ; Otago Witness, $\mathrm{n}^{\circ} 576,13$ décembre 1862; The Courier (Brisbane, Qld, 1861-1864), 29 novembre 1862.

\section{RÉSUMÉS}

La Grande-Bretagne, au moment même où elle connaît son apogée comme puissance coloniale, est, au $\mathrm{XIX}^{\mathrm{e}}$ siècle, la région d'Europe qui contribue le plus à l'émigration, principalement à destination de l'Australie, de la Nouvelle-Zélande, du Canada et des États-Unis. Si cette émigration est, au départ, principalement masculine, la proportion de femmes ne va cesser de croître, encouragée et assistée, à partir des années 1840, par un certain nombre d'associations philanthropiques. Il ne s'agit pas encore, à ce stade, de servir un objectif ouvertement impérialiste mais plutôt de répondre aux besoins des colonies tout en résolvant certains problèmes domestiques liés à la pauvreté ainsi que, de plus en plus, à l'excédent de femmes par rapport aux hommes révélé par les recensements successifs. L'évolution dans la façon de percevoir cette émigration, envisagée dans un premier temps comme solution à la pauvreté puis, à partir du recensement de 1851, comme remède au déséquilibre démographique dont semble souffrir le pays, donne alors lieu à une succession, mais également parfois à une juxtaposition de projets pouvant être qualifiés de philanthropiques, antiféministes ou féministes. Durant cette période charnière en matière d'émigration féminine, qu'il s'agisse de la catégorie sociale des femmes visées, des objectifs à atteindre, ou encore des moyens à employer, ces discours révèlent toutefois de nombreuses hésitations, voire contradictions, qui vont principalement s'expliquer par la difficulté, pour leurs auteurs, de concilier pragmatisme et idéologie.

Great Britain, while enjoying its peak as a colonial power was, in the nineteenth century, the region of Europe that contributed most to emigration-mostly to Australia, New Zealand, Canada, or the United States. Although this emigration was mostly male to begin with, the proportion of women grew from the 1840s onwards under the impulse, and with the support, of a number of philanthropic associations. The aim was not yet primarily to serve an imperialist agenda but rather to answer the colonies' demands in terms of labour and wives while solving at the same 
time domestic issues related to poverty as well as to the perceived surplus of single women revealed by the successive national censuses. The changing perception of female emigrationregarded first as a way to improve the condition of poor female workers then, following 1851, as a remedy to the demographic imbalance between men and women from which the country seemed to be suffering-was accompanied by a succession as well as, occasionally, a juxtaposition of schemes of a more or less philanthropic, antifeminist or feminist nature. During this pivotal period, partly due to the difficulty in reconciling pragmatism and ideology, hesitations and even contradictions emerge from the discourses held by their proponents, concerning the social category of women to be targeted, the goals to be achieved and the means to do so.

\section{INDEX}

Mots-clés : femmes, émigration, Grande-Bretagne, dix-neuvième siècle, colonisation

Keywords : women, emigration, Britain, nineteenth century, colonisation

\section{AUTEUR}

\section{VÉRONIQUE MOLINARI}

Université Grenoble Alpes, ILCEA4.

Véronique Molinari est agrégée et professeur des universités en civilisation britannique à l'Université Grenoble Alpes. Sa recherche porte sur le mouvement britannique victorien et édouardien ainsi que sur la participation des femmes en politique. Elle a publié de nombreux articles sur la question ainsi que plusieurs ouvrages, dont : Citoyennes, et après? Le droit de vote des femmes et ses conséquences en Grande-Bretagne, 1918-1939 (Peter Lang, 2008), Women and Science (avec Donna Spalding Andréolle, Cambridge Scholars Publishing, 2011), Droits des femmes, droits des autres : des féministes britanniques face aux autres exclus de la citoyenneté (1860-1930) (Presses universitaires Blaise Pascal, 2012) et Using and Abusing Science (avec Cyril Besson, 2016). veronique.molinari@univ-grenoble-alpes.fr 